\title{
Real-time ultrasound guided thoracic epidural catheterization: a technical review
}

\section{Jong-Hyuk Lee, Doo-Hwan Kim, and Won Uk Koh}

Received June 7, 2021

Accepted June 14, 2021
Department of Anesthesiology and Pain Medicine, Asan Medical Center, University of Ulsan College of Medicine, Seoul, Korea

\author{
Corresponding author \\ Won Uk Koh, M.D., Ph.D. \\ Department of Anesthesiology and \\ Pain Medicine, Asan Medical Center, \\ University of Ulsan College of \\ Medicine, 88 Olympic-ro 43-gil, \\ Songpa-gu, Seoul 05505, Korea \\ Tel: 82-2-3010-5606 \\ Fax: 82-2-3010-6790 \\ E-mail: koh9726@naver.com
}

\begin{abstract}
Thoracic epidural analgesia is known to have superior perioperative pain control over intravenous opioid analgesia in open abdominal surgery and is an essential enhanced recovery after surgery component in major abdominal surgeries. Recently, the ultrasound-guided thoracic epidural catheter placement (TECP) technique has drawn attention as an alternative for the traditional landmark palpation-based TECP or fluoroscopic-guided TECP technique due to the equipment's improvement and increased popularity. However, only a small number of studies have introduced the advantages and usefulness of ultrasound-guided TECP. Moreover, a certain level of ultrasound-guided in-plane technique is required to perform this technique. Thus, to apply ultrasound-guided TECP correctly and reduce the likelihood of side effects and complications, the practitioner must have a thorough understanding of the anatomical region, optimal block positioning, device selection, and management. In this technical review, the authors have compared the advantages and disadvantages of ultrasound-guided TECP to traditional techniques and described its technical aspects from patient positioning, ultrasound probe selection and scanning, needle insertion under ultrasound guidance, and successful thoracic epidural catheter insertion confirmation through ultrasound imaging. Additionally, the recommended epidural catheter tip placement level with the extent of its injectate epidural spread is further described in this review in reference to a recent prospective study published by the authors.
\end{abstract}

Keywords: Catheterization; Epidural analgesia; Thoracic vertebrae; Ultrasonography.

\section{INTRODUCTION}

Thoracic epidural analgesia (TEA) is known to have superior perioperative pain control over intravenous (IV) opioid analgesia in open abdominal surgery [1]. In fact, previous studies [2,3] showed that continuous TEA reduced incidences of postoperative gastrointestinal dysfunction and opioid consumption as compared to IV opioid analgesia, resulting in less postoperative sedation complications following intra-abdominal surgeries [4]. In addition to providing effective perioperative pain control, continuous TEA was found to reduce intraoperative surgical stimulation, which resulted in reduced insulin resistance and neuroendocrine stress response, further contributing to intraoperative protein catabolism. Therefore, body mass attenuation after surgery is reduced in postsurgical patients, helping in the improvement of postoperative outcomes in sarcopenic and frail patients $[5,6]$.

Traditionally, continuous TEA implementation has been described using two approaches - landmark palpation-based thoracic epidural catheter placement (TECP) technique or fluoroscopic-guided TECP technique. Although both tech-

This is an Open Access article distributed under the terms of the Creative Commons Attribution Non-Commercial License (http://creativecommons.org/licenses/by-nc/4.0) which permits unrestricted non-commercial use, distribution, and reproduction in any medium, provided the original work is properly cited.

Copyright (C) the Korean Society of Anesthesiologists, 2021 
niques have been widely accepted and used for a long time in clinical practice, they also have well-known drawbacks. Landmark palpation-based TECP, in particular, is associated with a relatively high failure rate $(12-40 \%)[7,8]$. On the other hand, fluoroscopic-guided TECP has a higher success rate than the former since it can accurately identify anatomical structures and epidural spaces using imaging devices [9]; however, due to the difficulty of using fluoroscopy in daily clinical practice and the burden of radiation exposure, its practical use is limited [10].

Recently, the ultrasound-guided TECP technique has drawn attention with the equipment's improvement and increasing popularity $[11,12]$. Despite this, only a small number of studies have introduced the advantages and usefulness of the technique $[8,13,14]$. Thus, in this technical review, the authors investigated the advantages of the ultrasound-guided TECP technique as compared to the other two. Thereafter, the technique's application for enhanced recovery after surgery in major surgeries was described based on the author's clinical experience.

\section{COMPARISON WITH OTHER TECHNICAL APPROACHES FOR THORACIC EPIDURAL CATHETER PLACEMENT}

The ultrasound-guided thoracic epidural approach provides further information on the spinal anatomical structure as compared to the landmark palpation-based technique. Utilizing ultrasound imaging of the spine before the neuraxial procedure will allow the preprocedural determination of the spinal level and depth up to the ligamentum flavum and posterior dura, as well as identify the anatomical abnormality, thus increasing its success rate [15-17]. A previous study by Salman et al has assessed the accuracy of preprocedural ultrasound scanning of the thoracic spine to measure the depth to the epidural space and determine the optimal puncture site for epidural needle placement of the thoracic spine. The authors found a good correlation of the distance to the epidural space between the ultrasound guided estimation and actual needle distance in total 33 study patients, demonstrating a Pearson correlation coefficient of 0.75 [17]. Additional advantages of this technique is that, it can further be applied to patients with significant degenerative spinal changes or had previously undergone spinal surgeries, wherein procedure performance and success rate are improved, as long as there is sufficient space for the needle to pass [16]. Furthermore, by using the real-time ultrasound-guided technique, the practic- ing physician can also monitor the target point location (ligamentum flavum or posterior epidural space) and epidural needle tip position in real time.

However, compared to the landmark palpation-based technique and preprocedural ultrasound imaging technique, a certain level of ultrasound-guided in-plane technique is required for real-time ultrasound-guided approach. Thus, sufficient experience and operation ability for ultrasound procedures is needed. Additionally, it is not easy to determine loss of resistance (LOR) with only one hand, while holding the probe with the other. Therefore, when it is visually confirmed that the epidural needle has touched the ligamentum flavum or upper end of the lamina, further entry and confirmation of the needle tip in the epidural space with LOR may be accomplished using the traditional blind technique. If any air enters the field during the procedure, an adequate image cannot be obtained, and thus saline should be used rather than air for LOR confirmation. Further technical descriptions are provided in the next section.

The greatest advantage of ultrasound-guided TECP as compared to fluoroscopy-guided TECP is that it avoids the burden of radiation exposure, which is a significant benefit for both patients and practicing physicians. Owing to the compact features of many modern ultrasound equipment, this becomes more feasible compared to the relatively bulky fluoroscopic machine, which is a spatial advantage in operation and anesthetic theaters with limited spaces. Another advantage is that the procedure is possible in any position, except in the supine position.

However, the disadvantage of the real-time ultrasoundguided technique over fluoroscopic-guided TECP is the difficulty in observing the catheter tip and drug epidural spread. Although visual confirmation of epidural lumbar spine catheterization and the posterior epidural spread of local anesthetics can be done by downshifting of the dura even in the ultrasound-guided technique, this differs at the thoracic level since the interlaminar space width varies from individual to individual, wherein the posterior dura is often not visible. Thus, in some cases, the catheter tip may exit through the transvertebral foramen, which may not be noticed until the patient's dermatome is checked postoperatively and is mainly due to different viewing directions performed intraoperatively (fluoroscopic-guided TECP, anteroposterior view; ultrasound-guided technique, sagittal view). Another disadvantage is the difficulty in performing the procedure again at the same spinal level should a small amount of air enter and distort the ultrasound view. 


\section{TECHNICAL DESCRIPTION OF REAL- TIME ULTRASOUND-GUIDED THORACIC EPIDURAL CATHETER PLACEMENT}

Real-time ultrasound-guided TECP can be performed in either prone, lateral, or sitting positions. However, without the aid of a positioning device or assistant, it can be difficult to maintain a fixed patient position while sitting. Therefore, the authors perform TECP in either the prone or lateral position, with more preference towards the prone position approach.

After entering the operation theater or preinduction room, basic monitors, including pulse oximetry, noninvasive blood pressure monitoring, and electrocardiogram, are applied to the patient. Following this IV access is performed, wherein a small amount of midazolam may be injected for patient comfort, but the procedure is generally performed without sedation. The patient is then placed in the prone position with a pillow under the upper abdomen to widen the interlaminar space. Traditionally, the interlaminar space between $\mathrm{T} 6$ and $\mathrm{T} 8$ has been recommended as a target space for epidural catheterization in patients undergoing upper abdominal surgery [18]. However, based on the clinical experience of two physicians ( $\mathrm{LJH}$ and $\mathrm{KDH}$ ) who have done this procedure at several different levels for more than two years, the T10-T11 interlaminar space is selected as the preferred target in our institute for real-time ultrasound guided TECP in order to place the catheter tip in the T9 vertebral level. The evidence for selecting the target level of the catheter tip is further described in the next section of the current article [12].

Before skin disinfection of the treatment area, an ultrasound prescan of the procedure area is done to check the target interlaminar space in the following order:

First, a high-frequency linear ultrasound probe is selected and placed in the longitudinal sagittal plane over the thoracic spinal column. In young children, the spinal column can be penetrated in part by ultrasound, and all or most parts of the relevant anatomical structures (spaces between the spinal processes, vertebral discs, ligamentum flavum, dura mater, intrathecal space, spinal cord, nerve roots, and nerve fibers) can be clearly distinguished using the linear probe [19]. In adults, a curved convex ultrasound probe is widely used in most of the previous reports when an epidural catheter is inserted under ultrasound guidance, especially in the lumbar regions [20-22]. However, when performing ultrasound-guided TECP, both linear and curved probes may be used. Since the thoracic spines are dorsally convex at shallow depths as compared the lumbar level spines, a linear probe is sufficient to identify the main object around the epidural space in majority of cases, unless the patient is very obese. This is supported by Pak and Gulati's previous study [13], which performed thoracic epidural catheterization in a similar manner, determining the mean parasagittal distance from the skin to the epidural space was $5.2 \pm 1.1 \mathrm{~cm}$. Aside from this, it is also helpful to use a linear probe when accurately checking the needle tips in real time is essential.

After confirming the level of the spine desired by the operator, the probe is placed on the midline to observe the spinous process (Fig. 1A-5, 6). This authors use the method of rib counting upwards after finding the 12th rib to check the level. Then, by sliding the probe in the laretal direction, the corresponding laminae can be confirmed (Fig. 1B-5, 6) In this state, the probe laterally tilts to obtain the paramedian sagittal oblique view. However, if tilting is insufficient or the intetlaminar space is too narrow, the superior articular process of the inferior vertebrae between the laminae may be seen (Fig. 1C-5, 6). At the lumbar level, an epidural needle can be advanced in this state, but at the thoracic level which has narrower interlaminar spaces, the operator can slightly tilt the probe laterally, making it possible to secure a paramedian sagittal oblique view with a wider interlaminar space (Fig. 1D-5, 6).

Consequently, the posterior complex (the ligamentum flavum and posterior dura) is observed as a linear hyperechoic structure between the laminae (Fig. 1D-5, 6), and the intrathecal space and spinal cord (anechoic), as well as the alternative complex (hyperechoic), can be visualized in this view. Moreover, the cephalad end of the probe can be further pivoted medially to decrease the laminal height at the level below (Fig. 1E-5, 6).

After checking the target interlaminar space through the ultrasound prescan, the patient's skin is marked and disinfected to sterilize the procedure area. After local infiltration with $2 \%$ lidocaine at the intended needle entry site, an 18-gauge Tuohy needle is inserted from the caudal end of the probe and advanced with in-plane view under real-time ultrasound guidance until the needle tip reaches the posterior complex in the target interlaminar space (Fig. 2). Needle-beam alignment can be maintained by advancing the needle in a similar lateral-to-medial trajectory. Furthermore, the probe and beam position should be held in constant to keep the target in view, in which the needle trajectory can be adjusted to keep the epidural needle visualized until the tip 

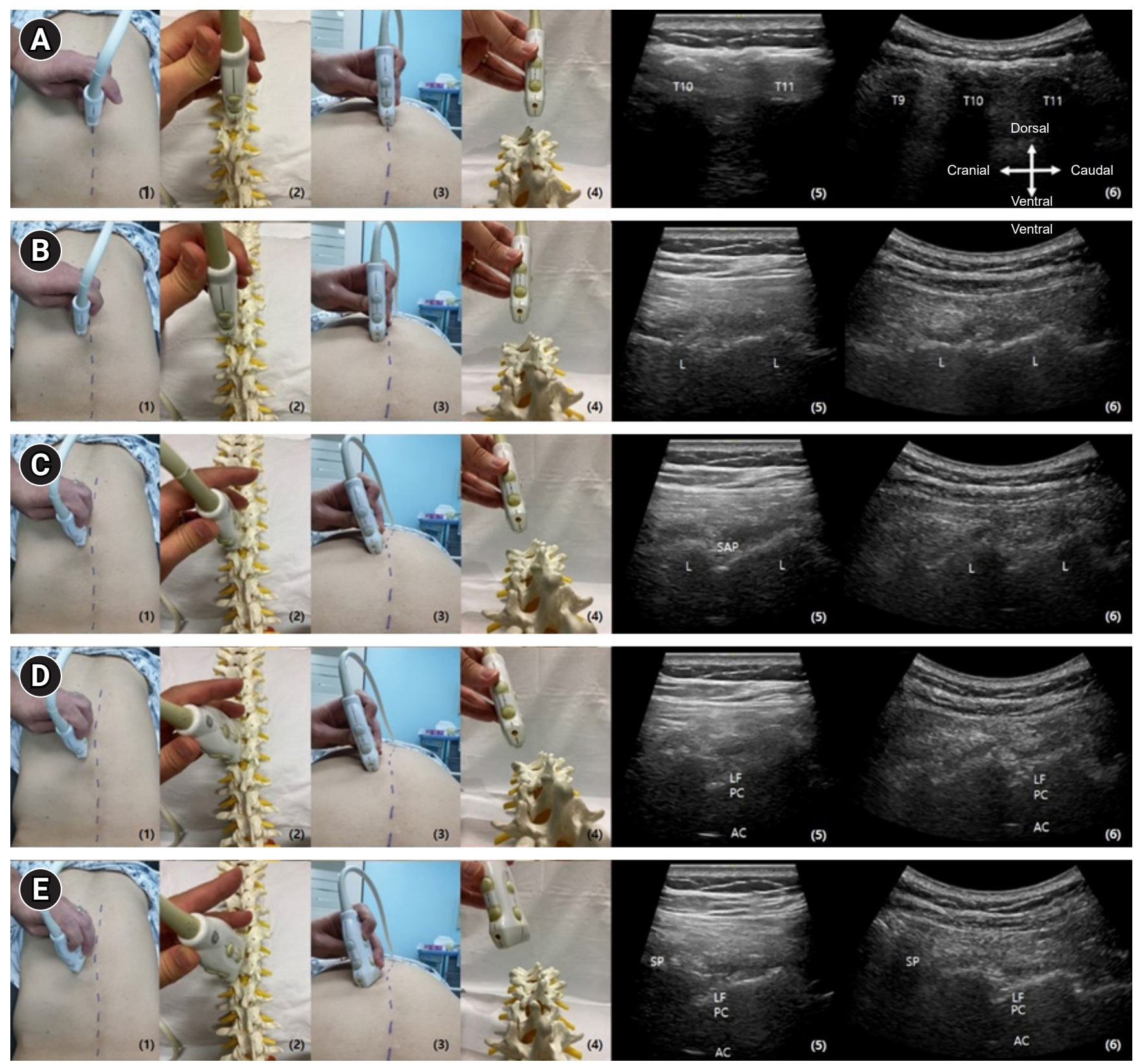

Fig. 1. Location of the ultrasound probe and the corresponding ultrasound views and its movements to obtain proper ultrasound views. (A) The probe positioned in midline to identify the spinous process (SP) of target spine level. (B) The probe moves laterally from the SP to obtain corresponding Laminae (L). (C) The probe laterally tilts to obtain the paramedian sagittal oblique view. $L$ resembling wave-like structures and superior articular process (SAP) of inferior vertebrae between the $L$ are seen in this view. (D) The probe slightly laterally tilts to obtain the paramedian sagittal oblique view. The ligamentum flavum (LF) and posterior dura of the posterior complex (PC) are seen as linear hyperechoic structures between the $L$ in this paramedian sagittal oblique view. If the interlaminar space is wide enough, anterior complex (AC) may be seen. (E) A decrease in the height of $L$ of the inferior vertebral body as compared to that in the paramedian sagittal oblique view is observed when the cephalad end of the probe is pivoted medially. (1) Position of probe and surface anatomy of the posterior thoracic level: posterior aspect view. (2) Position of probe for image 1 on the artificial spine model. (3) Position of probe and surface anatomy of the posterior thoracic level: inferior aspect view. (4) Position of probe for image 3 on the artificial spine model. (5) Ultrasound image of linear probe. (6) Ultrasound image of curved probe.

is located just in front of the posterior complex. If the needle tip is not visible, tilting the probe to the medial and lateral sides while shaking it slightly helps to determine its position, which is a method described in Gnaho et al.s study [14] for real-time ultrasound-guided epidural catheter insertion in obese parturients and can be usefully applied in other types 


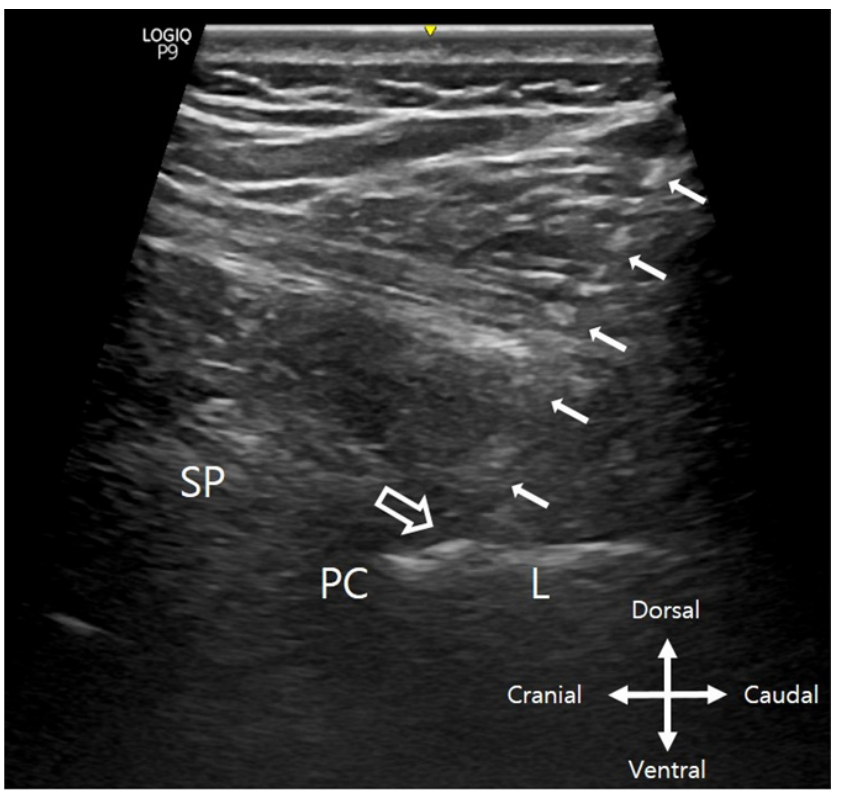

Fig. 2. Real-time ultrasound views for needle. PC indicate the posterior complexes. The arrow and open arrow indicate the epidural needle and the needle tip, respectively. PC: posterior complex, SP: spinous process, L: laminae.

of real-time ultrasound-guided procedures. Simultaneously, the LOR also should be checked, but if it cannot be done using only one hand, this can be further checked with a blind technique using both hands with normal saline from the point after the needle tip reaches the posterior complex in the ultrasound view. At this point, the performing physician should secure the needle with the operating hand to avoid needle tip position changes and further advance in the desired direction. After needle entry confirmation into the thoracic epidural space using saline LOR, the epidural catheter is advanced through the needle such that $3-5 \mathrm{~cm}$ of the catheter remained in the epidural space. After epidural catheterization, a small amount of test drug or saline injection via the epidural catheter will further help to confirm its placement. If the catheter is outside the epidural space, bulging around the lamina can be observed, and if the catheter is within it, visualization of the posterior dura downward shifting in the ultrasound image can possibly be observed. In a previous study, air was used to check the LOR [13]. However, in our experience, if air is used for LOR, it may be difficult to observe the downward shifting of the posterior dura when catheterization is successful or the bulging around the lamina when it fails due to the ultrasound image artifact caused by air insertion. Moreover, it becomes difficult to retry the procedure at the same level when it is required due to this obstruction.

\section{ACCURACY OF REAL-TIME ULTRASOUND GUIDED THORACIC EPIDURAL CATHETERIZATION}

Regardless of how well the catheterization is performed, if a segmental block cannot be confirmed, the practicing physician may not be confident about the epidural patient-controlled analgesia (PCA) effectiveness. Therefore, epidural spreading identification is of clinical importance. Under the ultrasound-guided technique, however, it is difficult to determine the exact degree of epidural spreading, although it is possible to determine whether an epidural spread was done. Thus, the author conducted a prospective study to determine epidural spreading by injecting a contrast agent under fluoroscopic guidance following ultrasound-guided catheterization [12].

This was recently conducted as a prospective study to identify fluoroscopic findings in patients who underwent a continuous thoracic epidural catheter using the real-time ultrasound-guided technique [12]. The average age of the 38 patients in this study was $62.5 \pm 9.9$ years, with $60.5 \%$ of them being male. The target interlaminar level for catheter entry in the epidural space was determined at either T9-10 or T10-11, and the average time it took to mark the skin for treatment was $49.5 \pm 13.8 \mathrm{~s}$, which took less time as compared to Auyong et al.'s [8] results that had an average time to mark spaces in $85 \mathrm{~s}$ and needling time of $188.5 \mathrm{~s}$. The reason for this difference was that the authors omitted to perform the midline checking procedure by changing the ultrasound probe to the transverse view during the prescan, which was also done in their current study. The median time for epidural needle placement was noted to be $49 \mathrm{~s}$ (IQR: 39-65 s), and there were 9 (23.7\%) cases of needle withdrawal with epidural needle direction changes, since the entry of the Tuohy needle into the epidural space was not successful at the first attempt, along with $2(5.3 \%)$ cases in which the needle direction was changed more than once. Overall, the probability of successful catheter insertion at the first attempt was similar to that in a previous study by Auyong et al. [8], which was approximately $70 \%$, and all patients in this study only underwent a single skin puncture for catheterization. The depth from the skin was mostly approximately 4 $\mathrm{cm}$, but since the epidural needle entered obliquely from the skin, the mean distance between the skin and epidural space was $5.6 \pm 0.5 \mathrm{~cm}$, which is comparable to Pak and Gulati's [13] previous results $(5.2 \pm 1.1 \mathrm{~cm})$.

After injecting contrast medium through the indwelling 
epidural catheter, the epidural spread was identified using a fluoroscope. All catheter tips were successfully placed in the epidural space, mostly at the level between T9 and T10 (n = $32,84.3 \%)$ and at the median epidural space ( $n=26,68.4 \%)$. After injecting the $4 \mathrm{ml}$ mixture of contrast medium, the mean cranial dispersion was noted to be in $5.4 \pm 1.6$ vertebral body levels mostly up to $\mathrm{T} 4$, with a mean caudal spread of $2.6 \pm 1.0$ vertebral body levels. In the cephalad direction, $94.7 \%$ of patients had an injectate spread of more than the T6 level, and 58\% presented with more than the T4 level. Meanwhile, in the caudal direction, $97.4 \%$ presented with a spread of more than the T11 level, and $28.9 \%$ of the patients went over the L1 level. These data provide further information on the epidural spread extent for adjustment of the hourly volume limit when an epidural PCA pump will be planned for postoperative analgesia.

\section{CONCLUSION}

In this review, we described the technical methods and tips of real-time ultrasound-guided thoracic epidural catheterization. It was found that ultrasound guidance during thoracic epidural catheterization reduced epidural needle placement time and the number of needle passes as compared to the landmark palpation technique [8]. Moreover, it gives more confidence to the practitioner as the needle tip position is visible throughout the procedure, making it possible to determine whether or not there was epidural spread [12]. Ultrasound guidance for epidural surgery has gained popularity and interest, especially for lumbar epidural needle placement and catheterization [23-25], and its application in thoracic epidural surgeries has also been receiving attention. However, studies describing its technical aspect, efficacy, and safety as compared to traditional techniques are still limited. Therefore, further studies to elucidate the advantages of ultrasound-guided thoracic epidural needles and catheter placement are essential in the future.

\section{CONFLICTS OF INTEREST}

No potential conflict of interest relevant to this article was reported.

\section{DATA AVAILABILITY STATEMENT}

Not applicable.

\section{AUTHOR CONTRIBUTIONS}

Conceptualization: Won Uk Koh. Data curation: JongHyuk Lee, Doo-Hwan Kim, Won Uk Koh. Formal analysis: Jong-Hyuk Lee, Won Uk Koh. Methodology: Jong-Hyuk Lee, Doo-Hwan Kim, Won Uk Koh. Project administration: JongHyuk Lee, Doo-Hwan Kim. Investigation: Jong-Hyuk Lee, Doo-Hwan Kim, Won Uk Koh. Resources: Won Uk Koh. Supervision: Won Uk Koh. Validation: Jong-Hyuk Lee, DooHwan Kim, Won Uk Koh. Writing - original draft: Jong-Hyuk Lee, Doo-Hwan Kim, Won Uk Koh. Writing - review \& editing: Jong-Hyuk Lee, Won Uk Koh.

\section{ORCID}

Jong-Hyuk Lee, https://orcid.org/0000-0003-0952-3900

Doo-Hwan Kim, https://orcid.org/0000-0002-3875-0857

Won Uk Koh, https://orcid.org/0000-0003-4881-1884

\section{REFERENCES}

1. Block BM, Liu SS, Rowlingson AJ, Cowan AR, Cowan JA Jr, Wu CL. Efficacy of postoperative epidural analgesia: a meta-analysis. JAMA 2003; 290: 2455-63.

2. Radovanović D, Radovanović Z, Škorić-Jokić S, Tatić M, Mandić A, Ivković-Kapicl T. Thoracic epidural versus intravenous patient-controlled analgesia after open colorectal cancer surgery. Acta Clin Croat 2017; 56: 244-54.

3. Aloia TA, Kim BJ, Segraves-Chun YS, Cata JP, Truty MJ, Shi Q, et al. A Randomized controlled trial of postoperative thoracic epidural analgesia versus intravenous patient-controlled analgesia after major hepatopancreatobiliary surgery. Ann Surg 2017; 266: 545-54.

4. Pöpping DM, Elia N, Marret E, Remy C, Tramèr MR. Protective effects of epidural analgesia on pulmonary complications after abdominal and thoracic surgery: a meta-analysis. Arch Surg 2008; 143: 990-9; discussion 1000

5. Schricker T, Lattermann R. Strategies to attenuate the catabolic response to surgery and improve perioperative outcomes. Can J Anaesth 2007; 54: 414-9.

6. Carli F, Halliday D. Continuous epidural blockade arrests the postoperative decrease in muscle protein fractional synthetic rate in surgical patients. Anesthesiology 1997; 86: 1033-40.

7. Hermanides J, Hollmann MW, Stevens MF, Lirk P. Failed epidural: causes and management. Br J Anaesth 2012; 109: 144-54.

8. Auyong DB, Hostetter L, Yuan SC, Slee AE, Hanson NA. Evaluation of ultrasound-assisted thoracic epidural placement in 
patients undergoing upper abdominal and thoracic surgery: a randomized, double-blind study. Reg Anesth Pain Med 2017; 42: 204-9.

9. Parra MC, Washburn K, Brown JR, Beach ML, Yeager MP, Barr $\mathrm{P}$, et al. Fluoroscopic guidance increases the incidence of thoracic epidural catheter placement within the epidural space: a randomized trial. Reg Anesth Pain Med 2017; 42: 17-24.

10. Kim DH, Lee MS, Lee S, Yoon SH, Shin JW, Choi SS. A prospective randomized comparison of the efficacy of ultrasound- vs fluoroscopy-guided genicular nerve block for chronic knee osteoarthritis. Pain Physician 2019; 22: 139-46.

11. Karmakar MK, Li X, Ho AM, Kwok WH, Chui PT. Real-time ultrasound-guided paramedian epidural access: evaluation of a novel in-plane technique. Br J Anaesth 2009; 102: 845-54.

12. Kim DH, Lee JH, Sim JH, Jeong W, Lee D, Kwon HM, et al. Real-time ultrasound-guided low thoracic epidural catheter placement: technical consideration and fluoroscopic evaluation. Reg Anesth Pain Med 2021; 46: 512-7.

13. Pak DJ, Gulati A. Real-time ultrasound-assisted thoracic epidural placement: a feasibility study of a novel technique. Reg Anesth Pain Med 2018; 43: 613-5.

14. Gnaho A, Nau A, Gentil ME. Real-time ultrasound-guided epidural catheter insertion in obese parturients. Obstet Anesth Digest 2016; 36: 161.

15. Grau T, Leipold RW, Delorme S, Martin E, Motsch J. Ultrasound imaging of the thoracic epidural space. Reg Anesth Pain Med 2002; 27: 200-6

16. Chin KJ, Karmakar MK, Peng P. Ultrasonography of the adult thoracic and lumbar spine for central neuraxial blockade. An- esthesiology 2011; 114: 1459-85.

17. Salman A, Arzola C, Tharmaratnam U, Balki M. Ultrasound imaging of the thoracic spine in paramedian sagittal oblique plane: the correlation between estimated and actual depth to the epidural space. Reg Anesth Pain Med 2011; 36: 542-7.

18. Miller RD, Eriksson LI, Fleisher LA, Wiener-Kronish JP, Cohen NH. Miller's anesthesia. 8th ed. Philadelphia, PA, Saunders. 2014.

19. Rapp HJ, Folger A, Grau T. Ultrasound-guided epidural catheter insertion in children. Anesth Analg 2005; 101: 333-9.

20. Ortega-Romero A, Domingo-Rufes T, del-Olmo C, Ismael MF, Mayoral V. Ultrasound-guided interventional procedures for lumbar pain. Tech Reg Anesth Pain Manag 2013; 17: 96-106.

21. Yamauchi M. Ultrasound-guided neuraxial block. Trends Anaesth Crit Care 2012; 2: 234-43.

22. Greher M, Moriggl B, Peng PW, Minella CE, Zacchino M, Eichenberger U. Ultrasound-guided approach for L5 dorsal ramus block and fluoroscopic evaluation in unpreselected cadavers. Reg Anesth Pain Med 2015; 40: 713-7.

23. Perlas A, Chaparro LE, Chin KJ. Lumbar neuraxial ultrasound for spinal and epidural anesthesia: a systematic review and meta-analysis. Reg Anesth Pain Med 2016; 41: 251-60.

24. Vallejo MC, Phelps AL, Singh S, Orebaugh SL, Sah N. Ultrasound decreases the failed labor epidural rate in resident trainees. Int J Obstet Anesth 2010; 19: 373-8.

25. Shaikh F, Brzezinski J, Alexander S, Arzola C, Carvalho JC, Beyene J, et al. Ultrasound imaging for lumbar punctures and epidural catheterisations: systematic review and meta-analysis. BMJ 2013; 346: f1720. 